better in time to satisfy the demand for a redefinition of the Metre. In the meantime frequency comparison chains are being developed at a number of National Laboratories that will provide sources of radiation in the optical region, phase locked to the Cs frequency standard, and therefore accurate to $\sim 10^{-13}$.

\title{
The Ryaberg
}

An improved measurement of the Rydberg has been made by Hansch and his colleagues at stanford in the U.S.A. by the use of polarization spectroscopy of the $3^{2} \mathrm{P}_{1 / 2}-2^{2} \mathrm{~S}_{1 / 2}$ line of Hydrogen (2). They obtained the value $\mathrm{R}_{\infty}=109737.31476(32)$ $\mathrm{cm}^{-1}$. Petley and Morris at N.P.L., England, have also made new measurements (3), finding $R_{\infty}=109737.3167(15) \mathrm{cm}^{-1}$, based on $3^{2} \mathrm{D}_{5 / 2}-2^{2} \mathrm{P}_{3 / 2}$ and $\mathrm{R}=109737.3179(15)$ $\mathrm{cm}^{-1}$ based on $3^{2} \mathrm{D}_{3 / 2}-2^{2} \mathrm{P}_{1 / 2}$ of Hyarogen.

\section{Secondary Standards}

In addition to the standards provided by lasers stabilized on absorption lines of $\mathrm{I}_{2}, \mathrm{CH}_{4}$ and $\mathrm{CO}_{2}$, mentioned above, radiations of comparable frequency reproducibility are produced by means of an Argon laser stabilized on $\mathrm{I}_{2}$ at $515 \mathrm{~nm}$ and a $\mathrm{CO}_{2}$ laser stabilized on lines of $\mathrm{SF}_{6}$ or $\mathrm{OSO}_{4}$ at $10 \mu \mathrm{m}$, but precise values for the wavelengths or frequencies have not yet been published. A He-Ne laser stabilized on an $I_{2}$ line at $612 \mathrm{~nm}$ has been measured at B.I.P.M., Paris, yielding a value for the wavelength $\lambda_{\mathrm{vac}}=611970458.6(.2) \mathrm{fm}$ (4). Laser sources such as these are becoming of increasing importance as references for the control of automatic wavelength measuring interferometers. Of the relatively little recent work on thermal sources of wavelength standards, the following is noteworthy: Norlén and Johansson at Lund in sweden have determined accurate values ( $\pm 0.001 \mathrm{~A}$ ) of $300 \mathrm{Fe}$ II lines between 2380 and $3000 \mathrm{~A}$ (5). Freeman and his group at N.P.I., England, are continuing work on U.V. lines of $\mathrm{Cu}$ II, measuring shifts, line widths and suitability for standards, but have not reported precise wavelength values. An atlas of 22,850 lines in the visible absorption spectrum of the $I_{2}$ molecule has been produced at the Aime-Cotton Laboratory in Paris (6). Internal consistency of $\pm 0.001 \mathrm{~cm}^{-1}$ and absolute accuracy of \pm 0.006 $\mathrm{cm}^{-1}$ are given. Tables of standard wave numbers for infrared spectroscopy have recently been published through the initiative of the Inter-Union Commission on Spectroscopy (7). A review article on wavelength standards will be included in Part B of Progress in Atomic Spectroscopy, soon to be published by Plenum Publishing $\mathrm{Co}$.

\section{REFERENCES}

1. 1976, Transactions I.A.U. 16A, Part 1, pp. 31-60.

2. Goldsmith, J.E. et al.: 1978, Priv. Comm., to be submitted to Phys. Rev. Lett.

3. Petley, B.W., Morris, K.: 1978, Priv. Comm.

4. Bennett, S.J. et al.: 1978, Priv. Comm., to be submitted to Metrologia.

5. Norlèn, G., Johannson, Se.: 1977, Annual Reports, University of Lund.

6. 1978, Atlas du Spectre d'Absorption de la Molecule d'Iode $14800-20000 \mathrm{~cm}^{-1}$, S. Gerstenkorn and P. Luc, Editions du CNRS, Paris.

7. 1976, Tables of Wave Numbers for the Calibration of Infrared spectrometers, 2nd ed., Ed. A.R.H. Cole, Pergamon, London.

K.M. BAIRD

Chairman of the Working Group

\section{WORKING GROUP 2: ATOMIC TRANSITION PROBABILITIES}

The Data Center on Atomic Transition Probabilities at the National Bureau of standards, washington, D.C., has continued its bibliographical and critical compilation work on transition probabilities. A new general bibliography has been 
published, covering the literature references through october 1977 (1). It contains approximately 2400 references in chronological order and includes listings by element, stage of ionization, and experimental or theoretical method applied, as well as an author index. A critical compilation has been completed for $\mathrm{V}, \mathrm{Cr}$, and Mn (2), which covers all stages of ionization on which reliable data are available. Work is now in progress on $\mathrm{Fe}, \mathrm{CO}$, and $\mathrm{Ni}$. A special NBS study has been devoted to oscillator strength distributions in various spectral series of li-like ions (3), and from this work extensive tables of critically evaluated oscillator strengths for the $\mathrm{Li}$ sequence up to $\mathrm{Ni}$ XXVI have been derived (4). A number of reviews on calculations and measurements of atomic transition probabilities and lifetimes have recently been published. The books "Beam-Foil Spectroscopy", edited by S. Bashkin (5) and "Progress in Atomic Spectroscopy", edited by W. Hanle and H. Kleinpoppen (6) contain several chapters on these topics. About 500 theoretical and experimental papers containing data on atomic transition probabilities have been published since 1976. Thus, rather than aim for completeness, a general review of the progress is given, and a large number of representative papers are briefly discussed. The review of the theoretical work is divided into two parts: First, papers which are included in the above cited general NBS bibliography are discussed without citation. Later on, the more recent papers are quoted with full references.

A number of the recent papers include data and/or formulae for many ions of a given isoelectronic sequence and as such are of interest in photospheric and coronal studies. Lin, Johnson, and Dalgarno (1977) have used the relativistic random-phase approximation to calculate transition probabilities for radiative decay from $\mathrm{n}=2$ states of He-like ions. Beck and Nicolaides (1976) have allowed for discrete- and continuum-configuration correlation in their variational treatment of ions of the $\mathrm{Li}, \mathrm{N}$, and F sequences. Kastner (1976) has used the Coulomb approximation to calculate the strengths of forbidden transitions for ions of the B, F, Al, and Cl sequences. Mewe (1977) has presented tables of semi-empirical parameters for transitions along isoelectronic sequences of $\mathrm{H}$ through $\mathrm{Al}$. Mühlethaler and Nussbaumer (1976) have used a scaled Thomas-Fermi potential with some configuration interaction for allowed and forbidden transitions of Be-like ions. Safronova (1976) has performed a charge-expansion perturbation calculation along the $\mathrm{N}$ sequence. Fischer (1976 and 1977) has applied multi-configuration Hartree-Fock theory to the calculation of oscillator strengths for the $\mathrm{Al}, \mathrm{Na}$, and $\mathrm{Cu}$ sequences. Armstrong, Fielder, and Iin (1976) have used multi-configuration Dirac-Hartree-Fock theory to predict f-values along the $\mathrm{Li}$ and Be sequences. Biemont (1977) calculated numerous transitions along the Li sequence by using a nonrelativistic Hartree-Fock approach. schrijver (1977) applied a hydrogenic approximation with intermediate coupling to the calculation of inner-shell transitions of Li-like ions, while Fox and Dalgarno (1977) have calculated additional inner-shell transitions along the same sequence by means of the z-expansion approach. Victor, Stewart, and Laughlin (1976) have used a semi-empirical model potential method with configuration interaction for low ions of the $\mathrm{Mg}$ and $\mathrm{Ca}$ sequences. Another group of papers contains data on highly ionized species of elements found in the solar corona. The HX (Hartree-Fock with statistical exchange) method of Cowan has been used by Bromage and Fawcett (1977) to calculate f-values of $\mathrm{C}-, \mathrm{N}-$, and $\mathrm{O}-\mathrm{like}$ ions of $\mathrm{Si}, \mathrm{K}, \mathrm{Ca}$, and $\mathrm{Fe}$, and Bromage, Fawcett, and Cowan (1977) studied Fe X, XI, XVIII, and XIX with this approach. Flower (1977) has calculated allowed and forbidden transitions of Fe XI and XII, and Dankwort and Trefftz (1976) have applied Multi-configuration Hartree-Fock theory to $\mathrm{Si} \mathrm{X}$. Several papers are devoted to the calculation of neutral and/or weakly ionized species. Saraph (1976) has applied the frozen-cores approximation to a few transitions of $\mathrm{Mg}$ II and to numerous ${ }^{1} \mathrm{~S}-{ }_{\mathrm{P}}$ transitions of neutral Mg. Biemont (1976) has applied a scaled Thomas-Fermi potential, and for some transitions a selfconsistent field approach, to the calculation of f-values of doubly ionized species of Sc through $\mathrm{Zn}$. Sinanoglu (1976) has reported results of his non-closed shell many-electron theory for transitions of many light atoms and ions. Bulatov (1976) has calculated A-values for microwave frequency transitions between fine-structure levels of the $2^{3} \mathrm{P}$ term of He $I$. 
The following theoretical papers are more recent and are therefore fully referenced. Iin, Johnson, and Dalgarno (7) have applied the relativistic randomphase approximation to several transitions of He-like ions. Shorer and Lin (8) utilized a Z-expansion approach to magnetic quadrupole transitions of the Be sequence, and Shorer has work in progress on the resonance lines of the $\mathrm{zn}$ isoelectronic sequence, in which he considers the effects of the $3 d$ subshell in particular. Lin, Laughlin, and Victor (9) have used model potential methods to calculate A-values of magnetic quadrupole transitions for $\mathrm{Be}$ - and $\mathrm{Mg}-\mathrm{like}$ ions. Relativistic HartreeFock f-values were calculated by $\mathrm{Kim}$ and Cheng (10) for ions of the Na sequence. Fischer and Hansen (11) applied multi-configuration Hartree-Fock theory to the resonance transition of the $\mathrm{zn}$ sequence, while Dankwort and Trefftz (12) applied the same method to the B sequence. Bromage, Cowan, and Fawcett (13) employed Cowan's HX method, including relativistic effects, for the calculation of f-values for Fe XII and XIII. Garstang, Robb, and Rountree (14) applied the close-coupling method with intermediate coupling to forbidaen transitions of $\mathrm{Fe} V I$, and Garstang reports further calculations in progress on TC I. Nussbaumer and Storey (15) used intermediate coupling to compute A-values for allowed and forbidden transitions of $C$ III. Nussbaumer has theoretical work in progress on a number of ions: N III, N IV, O V, F V, FVI, Ne VII, Ca XVII, Fe XXIII, Kr XXXIII, and Mo XXXIX. Dankwort (16) applied multi-configuration Hartree-Fock theory to the intercombination resonance line of Mg I. Pradhan and Saraph (17) calculated numerous oscillator strengths for $O$ I using a configuration-interaction approach. At Brussels, van Rensbergen reports calculations in progress on C II, C III, and C IV, based on scaled ThomasFermi wave functions.

The following review of the recent experimental work is organized according to the techniques applied. Only the very recent papers not covered by the April 1978 NBS bibliogxaphy axe referenced. Numerous direct measurements of transition probabilities have been performed by the traditional emission, absorption, and "hook" (anomalous dispersion) techniques. By means of emission measurements with wallstabilized arcs, Wujec and Weniger (18) and Wujec and Musielok (1976) determined absolute oscillator strengths for prominent lines of $\mathrm{Sn} I$ and II, as well as for some Cl I lines. Kühne, Danzmann, and Kock (19) used a wall-stabilized arc to measure $56 \mathrm{Ti}$ I lines. They combined their emission measurements with hook measurements. Czernichowski, Holys, and Roberts (1977) determined relative f-values for 27 ultraviolet $\mathrm{Ne} I$ lines with a wall-stabilized arc, and Ernst and schulz-Gulde (20) performed a similar experiment for $23 \mathrm{Kr}$ I Iines. A special stabilized arc source was used by Lotrian, Cariou, and Johannin-Gilles (1976) to study 17 uv lines of $\mathrm{Sn} I$, and Mosburg and Wilke (21) used a mercury discharge to determine the transition probabilities of $70 \mathrm{Hg}$ I lines spread over a wide wavelength range. A number of measurements have been done by a simplified emission technique, the branching ratio method, in which the lines originating from a common excited atomic level are studied. The requirements on the light source are minimal; however, the relative transition probabilities obtained for these small sets of lines must be normalized by a lifetime measurement for the level in question (which has usually been obtained from beam-foil spectroscopy). In particular, Whaling, Scalo, and Testerman (1977), Lage and Whaling (1976), and Maier and Whaling (22) have applied such a combination of measurements to determine transition probabilities of $T i I$, Pr II, and Nd II. Martinson, Curtis, Smith, and Biemont (23) used this technique on Mn II, Semenova and Smirnov (24) on Hg I, and Smirnov, Tsygir, and Yakovitskii (25) on neutral iodine. Using an advanced absorption technique, Blackwell and collaborators at oxford are continuing their high precision measurements of relative oscillator strengths of neutral iron. More than 100 relative f-values for transitions arising from low excitation states have been measured with a precision as high as 18 , and a report is in preparation. Other recent absorption measurements are those of Bieniewski (1976) on $\mathrm{Cr} I$, Bell and Lyzenga (1976) on Sc I (employing the atomic beam approach), and Hannaford and McDonald (26) on $\mathrm{Cu} I$. Huber and Sandeman (27) used a combined approach of absorption and hook measurements to study 148 transitions of neutral chromium, with an overlap of the two approaches for some 
lines of moderate strength. Hook measurements were performed by Parkinson, Reeves, and Tomkins, (1976) on Ca I, Sc I, Sr I, and Ba I, and by Smith and kühne (28) on $\mathrm{Ti} I$. Penkin and Komarovskii (1976) concentrated their hook measurements on neutral spectra of rare earth elements $\mathrm{Nd}, \mathrm{Sm}, \mathrm{Eu}, \mathrm{Gd}, \mathrm{DY}, \mathrm{Tm}$, and $\mathrm{Yb}$.

Many transition probabilities have been determined by measurements of the lifetimes of excited atomic levels. Due to the vigorous pursuit of beam-foil spectroscopy and due to the selective excitation capabilities of tunable lasers, this is currently a very active research area, with nearly 200 publications during the last three years, roughly half of them from beam-foll spectroscopy. Atomic lifetimes have been increasingly utilized to provide absolute scales for relative transition probability measurements. The high precision often obtained in lifetime measurements sometimes reaching the order of one percent, ties down absolute scales very accurately. The principal centres of lifetime measurements by beam-foil spectroscopy are the Universities of Aarhus, Alberta, Bochum, Kansas, Lund, Lyon, Quebec, Stockholm, Tennessee (Knoxville), Toledo, and Arizona (Tucson), and at the Argonne (Chicago), Darmstadt, and Berkeley laboratories. The new results are too numerous to be mentioned here individually. Instead, we note that many important new beam-foil results are covered in the Proceedings of the IVth and Vth International Beam-Foil Spectroscopy Conferences in Gatlinburg, Tennessee, 1975, and in Lyon, 1978, respectively. Furthermore, in the reviews by curtis (5), Cocke (29), and Marrus (5) representative samples of recent results are discussed. In the usual beam-foil experiment, cascade effects are often encountered due to the non-selective excitation of atomic levels, which causes the measured lifetimes to be too 10 ng. A theoretical simulation study by Younger and wiese (30) indicates that cascading effects are especially serious for $\Delta \mathrm{n}=0$ resonance transitions of heavy ions. cascading is eliminated by the beam-laser approach. Two-stage excitation results from beam-gas cell and subsequent beam-laser interaction. The laser excitation is tuned to specific transitions. Schulze-Hagenest et al. (1977) determined the lifetime of the Li I $3 d^{2} \mathrm{D}$ level to within one percent with such a technique. Similarly, Arnesen et al. (31) determined some lifetimes of La II, again with very high precision. In conventional lifetime experiments, selective excitation with tunable lasers is also increasingly applied to eliminate the cascading effects associated with electron beam excitation. For example, Marek and Vogt (1977), Marek (32), Marek and Münster (33), and Hotop and Marek (34) used a pulsed dye laser for excitation and employed the delayed coincidence technique to analyze the decay curves from a number of Co I, CS I, Sm I, and DY I excited atomic states, work on Fe I is in progress. Teppner and zimmermann (35) used an analogous technique to study some atomic levels of $\mathrm{K} I$ and $R b I$, and Bulos et al. (36) applied it to some states of La I. A number of lifetimes were also measured directly by observing the exponential intensity decays from states selectively populated by one- or two-step dye laser excitation. Havey, Balling, and Wright (1976) studied lifetimes in $\mathrm{Mg} I, \mathrm{AI} I, \mathrm{Ga} I$, Sr I, In I, TI I, and Ba II; Gornik (37) measured some higher-lying levels in the Sr I spectrum, and selter and kunze (38) directly recorded the decay curves of some $A I I$ and Ag I levels. Shaw, King, and Adams (39) determined lifetimes in $Z n I$ and II with another version of the delayed coincidence technique, which also circumvents cascading effects. Still another refined version of the delayed coincidence method is the high frequency deflection technique, with which Brzozowski et al. (1976) determined a number of $\mathrm{Fe} I$ and II lifetimes, and Bromander et al. (40) measured lifetimes in C I, N I, and O I. An additional experimental method which has recently been applied is the Hanle effect (or zero-field level crossing) technique for measuring atomic lifetimes; for example the measurements by Kelly and Mathur (1976) on Sr I, by Bulos, Gupta, and Happer (1976) on K I, Rb I, and Cs I; by Rambow and Schearer (1976) on Mg II, Ca II, Zn II, Sr II, Cd II, Ba II, and Yb I and II (utilizing Penning ionization in a fast-flowing helium afterglow to generate the ions); by Becker, Bucka, and Schmidt (1977) on $\mathrm{Cr} \mathrm{I}_{i}$ by Klotz, Becker, and Goebel (41) on Co I; by Landais, Chantepie and Laniepce (42) on $\mathrm{Zn} I$; and by Andersen, Poulsen, and Ramanujam (1976) on $\mathrm{Zn} \mathrm{II,} \mathrm{Cd} \mathrm{II,} \mathrm{and} \mathrm{Hg} \mathrm{II.} \mathrm{The} \mathrm{phase-}$ shift lifetime measurement technique has been applied by smith (43) to resonance transitions of F I, Si II, S I-III, and P II. Other specialized lifetime 
measurements have been reported by Nowak, Borst, and Fricke (44) on 0 I, using a time-of-flight technique, and by Ramanujam (45), using a sputtering technique to study some levels of $\mathrm{Fe} I, \mathrm{~Pb} I, \mathrm{Zr} I$, and $U \mathrm{I}$. Finally, it should be noted that Corliss and Tech (1976) have revised their extensive table of $\mathrm{Fe} I$ lifetimes on the basis of comparison with numerous recently measured values.

\section{REFERENCES}

1. Fuhr, J.R., Miller, B.J., Martin, G.A.: 1978, Bibliography on Atomic Transition Probabilities (1914 through October 1977), Nat. Bur. Stand. (U.S.) Spec. Publ. 505, U.S. Government Printing office, Washington, D.C.

2. Younger, S.M., Fuhr, J.R., Martin, G.A., Wiese, W.I.: 1978, J. Phys. Chem. Ref. Data 7, p. 495.

3. Martin, G.A., Wiese, W.L.: 1976, Phys. Rev. A 13, p. 699.

4. Martin, G.A., Wiese, W.I.: 1976, J. Phys. Chem. Ref. Data 5, p. 537.

5. Bashkin, S. (Ed.): 1976, Beam Foil Spectroscopy, Springer Verlag, Berlin.

6. Hanle, W., Kleinpoppen, H. (Eds.): 1978, Progress in Atomic Spectroscopy, Vols. I \& II, Plenum Press, New York.

7. Lin, C.D., Johnson, W.R., Dalgarno, A.: 1977, Astrophys. J. 217, p. 1011.

8. Shorer, P., Lin, C.D.: 1977, Phys. Rev. A 16, p. 2068.

9. Lin, C.D., Laughlin, C., Victor, G.A.: 1978, Astrophys. J. 220, p. 734.

10. Kim, Y. - K., Cheng, K.-T.: 1978, J. Opt. Soc. Am. 68, p. 836.

11. Fischer, C.F., Hansen, J.E.: 1978, Phys. Rev. A 17, p. 1956.

12. Dankwort, W., Trefftz, E.: 1978, Astron. Astrophys. 65, p. 93.

13. Bromage, C.E., Cowan, R.D., Fawcett, B.C.: 1978, Mon. Not. Roy. Astr. Soc. 183, p. 19.

14. Garstang, R.H., Robb, W.D., Rountree, S.P.: 1978, Astrophys. J. 222, p. 384 .

15. Nussbaumer, H., Storey, P.J.: 1978, Astron. Astrophys. 64, p. 139.

16. Dankwort, W.: 1977, J. Phys. B: At. Mol. Phys. 10, p. 3617.

17. Pradhan, A.K., Saraph, H.E.: 1977, J. Phys. B: At. Mol. Phys. 10, p. 3365.

18. Wujec, T., Weniger, S.: 1977, J. Quant. Spectr. Rad. Trans. 18, p. 509.

19. Kühne, M., Danzmann, K., Kock, M.: 1978, Astron. Astrophys. 64, p. 111.

20. Ernst, W.E., Schulz-Gulde, E.: 1978, Physica 93C, p. 136.

21. Mosburg Jr., E.R., Wilke, M.D.: 1978, J. Quant. Spectr. Rad. Trans. 19, p. 69.

22. Maier, R.S., Whaling, W.: 1977, J. Quant. Spectr. Rad. Transf. 18, p. 501.

23. Martinson, I, Curtis, L.J., Smith, P.L., Biémont, E.: 1977, Phys. Scr. 16, p. 35 .

24. Semenova, I.V., Smirnov, Yu.M.: 1977, opt. Spectrosc. 42, p. 477.

25. Smirnov, V.V., Tsygir, O.D., Yakovitskii, S.P.: 1977, Opt. Spectrosc. 43, p. 600 .

26. Hannaford, P., McDonald, D.C.: 1978, J. Phys. B: At. Mol. Phys. 11, p. 1177.

27. Huber, M.C.E., Sandeman, R.J.: 1977, Proc. Roy. Soc. A 357, p. 355.

28. Smith, P.L., Kühne, M.: 1978, Proc. Roy. Soc. A 362,' p. 263.

29. Cocke, C.L.: 1976, Beam Foil Spectroscopy, in "Methods of Experimental PhysicsSpectroscopy",Vol. 13, Part B (Ed. D. Williams), p. 213, Academic Press, New York.

30. Younger, S.M., Wiese, W.L.: 1978, Phys. Rev. A 17, p. 1944.

31. Arnesen, A., Bengtsson, A. Hallin, R., Lindskog, J., Nordling, C., Noreland, T.: 1977, Phys. Scr. 16, p. 31.

32. Marek, J.: 1977, J. Phys. B: At. Mol. Phys. 10, P. I325.

33. Marek, J., Münster, P.: 1978, Astron. Astrophys. 62, p. 245.

34. Hotop, R., Marek, J.: 1978, Z. Physik A 287, p. 15.

35. Teppner, U., Zimmermann, P.: 1978, Astron. Ástrophys. 64, p. 215.

36. Bulos, B.R., Glassman, A.J., Gupta, R., Moe, G.W.: 1978, J. Opt. Soc. Am. 68, p. 842 .

37. Gornik, W.: 1977, z. Physik A 283, p. 231.

38. Selter, K.P., Kunze, H.-J.: 1978, Astrophys. J. 221, p. 713.

39. Shaw, D.A., King, G.C., Adams, A.: 1978, J. Phys. B: At. Mol. Phys. 11, p. 239.

40. Bromander, J., Duric, N., Erman, P., Larsson, M.: 1978, Phys. Scr. 17, p. 119. 
41. Klotz, W.D., Becker, U., Goebel, L.H.: 1977, Astron. Astrophys. 61, p. 51.

42. Landais, J., Chantepie, M., Laniepce, B.: 1977, Opt. Commun. 23, p. 80.

43. Smith, W.H.: 1978, Phys. Scr. 17, p. 513.

44. Nowak, G., Borst, w.L., Fricke, J.: 1978, Phys. Rev. A 17, p. 1921.

45. Ramanujam, P.S.: 1977, Phys. Rev. Lett. 39, p. 1192.

\author{
W.L. WIESE \\ Chairman of the Working Group
}

\title{
WORKING GROUP 3: COLLISION CROSS-SECTIONS AND LINE BROADENING
}

\section{A. Line Broadening}

Stark broadening (1) and polarisation shift effect in high density plasmas (2) have been reviewed. Critical reviews and a tabulation of selected data of the Stark widths and shifts of lines of non hydrogenic atoms and ions have been published $(32,33)$.

1. Broadening and shift of spectral lines of hydrogen and hydrogenic ions in plasmas.

Interest in stark broadening of hydrogenic lines arising from dense, high temperature plasmas (2) - (11) is increasing for several reasons; one of them is the possibility of doing an experimental study of the far wings: this has enabled to evince predicted satellite structure (10). At high and low densities, the increase of experiments concexning the study of the center of the lines and the neighbouring wings have given convergent results (9), (11), (12), (13), (19) concerning the disagreement with theory. The importance of ion dynamics seems now well established: earlier experiments showing the dependence of the central dip of $H \beta$ on the reduced mass have been confirmed (12), (13). Yet the various theoretical attempts of calculation have all failed (14) - (16), with the exception of the MMM (model microfield method), which enables an uniform semi-classical unified treatment of electrons and ion perturbers (17), (25): strong effects are found in the line centers of Lya, Ha and $\mathrm{HB}$ which agree with earlier experiments but not with the most recent ones (20). Another Stark broadening mechanism has been suggested for taking into account the mass effect (3). The importance of the interference term is still being debated (21), (22) and fine structure effects have been pointed out (12), (23).

The theory of the line wings of hydrogen lines has made great progress, with particular attention paid to the Ly $\alpha$, LYB, H $\beta$ asymmetry (24) - (31). A detailed quantum calculation of the electronic broadening with a "dissection" of the various contribution has been made (24) - (26): in the far wings of Ly $\alpha$ electrons are predominant, short range and quantum effects are important; exchange is of drastic importance as well as quenching effects (27): this leads to the conclusion that the Holtsmark limit is a fiction. In the near wings ions become important and an improved semi-classical approximation including dipole, quadrupole and quadratic (polarization) interaction gives a correct asymmetry for Lya but not for Ly $\beta$ when compared to the experiment (31). For $H B$ the asymmetry calculated with the dipolar exact resonance theory (28) is in agreement with (11).

2. Broadening and shift of non hydrogenic atoms and ions in plasmas.

For isolated lines, experimental results of the two past years (34) - (46) (Ca II, Na I, He I, N I, C I, Si II, Al I, Al II, alkaline earth, Si III, IV, Ne II, F II, CI III, Ar II, U V) have confirmed the success of the semi-empirical and semiclassical impact perturbation theories. The unitarized quantum distorted wave calculation (45) agrees with the semi-classical one (39). This confirms the fact that the high order terms included in the determination of the perturber's trajectory are of minor importance (47). The MMM has been applied to isolated lines (48), (53) and agrees with earlier impact theories. 